\title{
Más allá delcapitalismocolonial: Diálogos y saberes socialistas como movimientos de resistencia en la educación
}

\begin{abstract}
Resumen: Una educación más allá delcapital significa-no sólo-resistir a los tratados colonial es impuestos por el legado europeo y la adopción de práctica simperialistas, sino también promover salidas, vías alternativas para formar otra sociedad, marcada por los valores e intereses que no son aliados del mercado de las grandes corporaciones y el status quo. La resistencia también está pensando en propuestas pedagógicas que cuestionan el orden capitalista que se inculca en los niños desde el nacimiento. La Revolución cubana, a pesar del bloqueo económico, comercial y financiero, priorizó la educación y hoy es un ejemplo de que la educación para todos, la calidad y la equidad.
\end{abstract}

Palabras clave: desigualdades. Educación cubana. Resistencia.

\section{Para além do capitalismo colonial: Diálogos e saberes socialistas como movimentos de resistência na educação}

Resumo: Uma educação para além do capital significa - não apenas - resistir aos tratados coloniais impostos pelo legado europeu e pelas adoções de práticas imperialistas, mas também de promover saídas, caminhos alternativos para a constituição de outra sociedade, marcado por valores e interesses que não estejam aliados com as grandes corporações do mercado e do status quo. Resistir também é pensar propostas pedagógicas que questionem a ordem capitalista que é incutida nas crianças desde o nascimento. A Revolução Cubana, apesar dos embargos econômicos, priorizou a educação e hoje é um exemplo de que é possível uma educação para todos/as com qualidade e equidade.

Palavras-chave: Desigualdade. Educação cubana. Resistência.

\section{Beyond colonial capitalism:Dialogues and socialist knowledge as resistance movements in education}

\begin{abstract}
An education beyond capital means - not only - to resist the colonial treaties imposed by the European legacy and the adoption of imperialist practices, but also to promote exits, alternative ways to form another society, marked by values and interests that are not allies Of the large corporations market and the status quo. The resistance is also thinking of pedagogical proposals that question the capitalist order that is instilled in children from birth. The Cuban Revolution, despite the economic, commercial and financial blockade, prioritized education and today is an example that education for all, quality and equity, is possible.
\end{abstract}

Keywords: Inequality. Cuban education. Resistance.

\footnotetext{
1 Mestre em Educação. Ministerio de Educación de la República de Cuba.E-mail: olga.franco@mined.rimed.cu
} 


\section{Introducción}

$\mathrm{E}$

l paso del a Edad Media a la de la modernidada los ojos del hombre europeo marca una ruptura conlas estructuras sociales, políticas y económicas que se desarrollan a través de las relaciones

feudales, y entre los factores que movilizaron a este conjunto de cambios, podemos destacar: la llegada de los españoles y portugueses a las Américasy la explotación de las riquezas de esta tierra, que culminaron en la intensificación comercial yel desarrollo del sistema capitalista.

Estos procesos históricos nos presentan cómo era posible- durante siglos - colonizarlos diferentes pueblos indígenas que vivíanen Estados Unidos, la personalización de las diferentes composiciones étnicas y de organización de un modelo cultural común, marcadas por limitaciones lingüísticas, sustento simbólico, religioso y económico es decir, la implantación del capitalismo como modo de producción de la riqueza y la segregación, en todos los ámbitos de la vida, con las jerarquías de clase, género, color de la piel, edad y otras diferencias causadas por la producción, reproducción, distribución y consumo. Se cree que el poder ideológico del capitalismo como un mecanismo educativo, que por medio de élse aprende adistinguir y estratificar los espacios, para dar prioridad alas relaciones humanas y tener o no acceso a los derechos humanos ganados duramente para una vida digna.

Las desigualdades económicas que afectan a todos los continentes y el imperialismo que explotay oprime principalmente a poblaciones enteras de los países del surdel mundo nos moviliza para cuestionar esta "educación para el capital", buscando en las contradicciones internas de este sistema para encontrar posibilidades de revoluciones, enfrentamiento a este orden de carácter global que no se limita sólo al aspecto económico, sino sobre todo cultural y político.

Una educación más allá del capital significa - no sólo - resistir a los tratados colonial es impuestos por el legado europeo y la adopción de prácticas imperialistas, sino también promover salidas, vías alternativas para formar otra sociedad, marcada por los valores e intereses que no son aliados del mercado delas grandes corporaciones y el status quo. La resistencia también está pensando en propuestas pedagógicas que cuestionan el orden capitalista que se inculca en los niños desde el nacimiento.

La Revolución cubana, a pesar del bloqueo económico, comercial y financiero, priorizó la educación y hoy es un ejemplo de que la educación para todos, la calidad y la equidad, es posible. Antes de 1959, el analfabetismo era del $23,6 \%$ y del $0,2 \%$ en la actualidad estos índices se lograron por el compromiso de toda la sociedad como es requerido por la Constitución de 1976 que el "estado socialista, como poder del pueblo [...] garantiza [...] no haya ni un niño sin escuela, sin alimentación y vestido, que todo joven tenga la oportunidad de estudiar;".

Una educación basada enla integridad humana requiere una formación que más allá del conocimiento técnico y científico también se desarrollan los valores de la responsabilidad, el trabajo (valor 
fundamental en una sociedad de trabajadores), y la solidaridad.A diferencia delmodelo capitalista en el que la subordinación y el consumo son los ejes principales de una educación alienada y alienante.

De esta forma, el énfasis en el menú de esta mesa se realiza desde el diálogo con experiencias educativas cubanas, atendiendo a los años de práctica, la investigación científica y la ideología socialista como movimientos de resistencia a las imposiciones liberales del mercado mundial.

\section{Algunas características de la educación en cuba desde la etapa colonial hasta nuestros días}

En Cuba la llegada de los colonizadores españoles no permitió el desarrollo natural, el propio Cristóbal Colón afirma que los indios (como él los llamó) tienen el mismo tipo físico: baja estatura, cráneo corto, pequeño, redondeado, nariz no muy ancha, su piel era de color oscuro rojizo, con poca barba, cabellos lacios, gruesos y muy negros y cuerpos bien proporcionados, todos entienden el idioma que hablan, el arahuaco, común en las Antillas, pero su diferencia está en el grado de desarrollo de la comunidad, en forma general eran muy sociables, de buen carácter, compartían juntos los Areítos, donde bailaban y les gustaba jugar a los Batos o pelotas.

Los Guanahatabeyes se consideran como los menos desarrollados ya que solo recolectaban lo necesario para vivir, le siguen en desarrollo los Siboneyes que se dedicaban a cazar y pescar y los más desarrollados eran los Taínos, que eran agricultores y ceramistas.

\section{Etapa colonial}

Abarca un largo proceso histórico de cerca de 390 años, con diferentes momentos evolutivos, en que Cuba fue realmente colonia de España en el más amplio sentido del concepto. El interés de la Metrópolis consistió siempre en españolizar al pueblo cubano, considerándolo parte de su país, después que la cubanía surgió como una realidad histórica a fines del s. XVIII e inicios del s. XIX. Se produjo entonces, una lucha por la conciencia entre las fuerzas españolas (oficiales) y los intereses del cubano (criollo), que deseaba defender su identidad y el desarrollo de la patria naciente, las leyes tan formales de España para el intercambio y pago de los productos crean un rechazo que va aumentando con la casi exterminación de los aborígenes y la trata de negros de África que vivían en condiciones infrahumanas, cuestión rechazada por los sectores más progresistas. La educación se convirtió en el campo de batalla por la ideología del cubano.

En casi 350 años, España se ocupó apenas de la educación de los niños de 0 a 6 años y solo haasta 1842 no estableció una Ley de Instrucción Pública para todo el país. No había educación primaria prácticamente y mucho menos interés por las edades más tempranas. Hay que decir, que en el mundo occidental tampoco existía esa preocupación como un principio y una necesidad, generalizadas.

Por razones "humanitarias" y por iniciativa privada o de instituciones religiosas se fundaron algunos centros para atender a los menesterosos, huérfanos o niños y niñas abandonados. Así fueron creadas 
instituciones, tales como: hospitales, casas para niños recién nacidos abandonados, casas cunas para los pequeños menores de años y la Casa de Beneficencia, triste recuerdo de esa época. Todo esto ocurrió entre 1603 y 1794.

Sin embargo, estas acciones no significaron en ninguna medida que se llevara a cabo una educación; no se reconoce en esta época la existencia de una concepción educativa propiamente dicha; lo que se les "educaba” era esencialmente de corte escolástico, y del tipo más retrógrado y recalcitrante, memorístico, sin tener en cuenta las características de los educandos.

A partir de 1790, comenzó a vislumbrarse una reacción, primero tímida y después más fuerte, contra el ideal hispano-escolástico, por los criollos, que necesitaban un cambio en la conciencia social.

\section{Había nacido la cubanía y era necesario defenderla a partir de la educación escolarizada.}

Aun que se apreciaba un mayor interés y atención por los niños menores de 6 años, por supuesto de una minoría, no se conoce que hubiese un criterio y una sensibilidad en cuanto a la educación en esas edades.

Es precisamente en el año 1861, en que se introdujo, por primera vez, en la Real Casa de Beneficencia, un ideal educativo coherente para los niños que hoy llamamos de "edades tempranas". Se introdujeron las ideas de Luz y Caballero y Félix Varela que representaba un pensamiento más moderno y una concepción pedagógica para la infancia temprana.

En 1880 se fundó en La Habana, el primer Kindergarten, lo que resulta un hecho importante, pues la institución dejaba de ser de beneficencia para convertirse en una institución educativa propiamente dicha, pero estas instituciones solo abarcaban a niñas y niños de 4 a 6 años.

José Martí, por estos tiempos plantea la necesidad de educar desde las edades tempranas, en el kindergarten, para que los niños tengan donde:

“... bañarse las manos, so pretexto de jugar en el agua con barquitos de papel, y donde habituarse al trabajo, orden y belleza, con el entretenimiento de los dibujos y tejidos, y donde elevar el espíritu con la música, y ennoblecerlo desde la raíz, con los tonos sentidos compuestos para los coros y la danza;..."

Las ideas Martí, en materia de atención a la infancia preescolar, no se aplicaron inmediatamente pero representaron un momento muy elevado, por cierto, de una concepción cubana y latinoamericana de la educación para los niños y las niñas de estas edades.

Si se detiene la reflexión en lo planteado en el pensamiento martiano, hay que percatarse que toda la orientación para la formación del niño en esas edades está implícita en las ideas siguientes:

- Empleo del juego para el desarrollo de la imaginación y el deleite en el niño.

- La orientación para el trabajo de gran valor educativo.

- La utilización del dibujo, la música, los coros y la danza, para la formación integral y estética del pequeño.

- La formación de valores desde esas tiernas edades.

Con esto queremos decir, que por primera vez, en el ideario educativo de Martí, aparece en Cuba una concepción original para la educación infantil.

Como hemos podido apreciar, en la etapa de la Colonia existió un largo período en que no se concibió una educación para los niños y niñas desde el nacimiento hasta su ingreso en la escuela primaria. La 
atención a los niños de estas edades se limitó a una ayuda piadosa para lograr la sobrevivencia de los huérfanos y abandonados. Esa atención respondió a criterios totalmente escolásticos y "españolizantes" desconociendo las particularidades del país y en contra de la formación de una verdadera identidad nacional.

En su etapa final se introdujeron las ideas e ideales educativos ya expresados por nuestros principales pedagogos: José de la Luz y Caballero, Félix Varela y nuestro José Martí, como fuertes opositores españoles.

La educación se caracteriza por ser diferenciada para aquellos que poseían riquezas y podían pagar una educación de calidad. Para los criollos pobres y algunos esclavos libertos otras eran las condiciones y para los esclavos no existían las posibilidades de recibir ninguna instrucción.

Se puede concluir sobre esta etapa que la educación respondía a los intereses de la clase que poseía el poder económico, político y social: los españoles y criollos ricos.

\section{Transición hacia el período de la república neocolonial (1899- 1902)}

Fue con la primera intervención norteamericana en Cuba, la cual se impuso, al robarle la independencia que tenían en sus manos los cubanos, que con su inoportuna e hipócrita intervención crearon una situación de riesgo para los cubanos y a partir de este período las condiciones tomaron un nuevo giro

En lo que respecta a la educación de los pequeños, en esta etapa se extendió el grado preescolar a todo el país de una manera oficial y se prepararon maestros con una cierta especialización para ello. Se abrieron "escuelas para párvulos", las que, debido a la carencia de locales, iniciaron sus actividades en las propias casas de las educadoras seleccionadas. La edad de los alumnos oscilaba entre los cinco y siete años.

Entre 1901 y 1902 se crearon las Escuelas Normales de Kindergarten para la formación de maestras en esta especialidad, con profesores y planes de estudios norteamericanos, que tenía la finalidad de introducir cambios en la educación cubana en concordancia con sus propios intereses. Se crearon, también por norteamericanos, algunos centros de kindergarten en distintas regiones del país, principalmente en las capitales de las provincias existentes en aquellos momentos.

Como se aprecia hasta aquí, la atención que se daba a la infancia cubana, y sobre todo, a la menor de seis años, se concentraba en las zonas urbanas, fundamentalmente, en la capital de la Isla y en algunas pocas capitales de provincias.

Los niños y niñas de zonas rurales sufrían completo desamparo y aún cuando comenzó la enseñanza en las escuelas en lugares donde vivían campesinos, estas no incluían en sus planes la atención a los niños en el período de 0 a 6 años.

En resumen, en este período, como resulta evidente, los Estados Unidos desarrollaron una ofensiva educativa en Cuba para norteamericanizar al cubano, al establecer un proyecto educativo más amplio, pero anexionista, con una dependencia teórica al ideal educativo de los Estados Unidos, por lo que es un sensible retroceso en la concepción educativa. 
En este escenario, durante las primeras décadas del siglo XX, coexistían instituciones tales como La Casa de Beneficencia, algunos asilos de huérfanos o niños abandonados, aproximadamente unas 30 creches y unos pocos establecimientos particulares.

En relación con las creches, su objetivo era el de ofrecer un consultorio para menores y la asistencia, el cuidado y la enseñanza de niños pobres. En ellas, un pequeño grupo de madres trabajadoras podía dejar a sus hijos e hijas entre las 6 a.m. y las 9 p.m. Estas creches, por lo general, albergaban de treinta a sesenta niños en locales inapropiados y faltos de higiene; el personal que las atendía no tenía ninguna preparación o eran prácticamente analfabetos. El régimen de vida que se aplicaba a los niños y las niñas era casi carcelario pues carecían de actividades apropiadas y atractivas, apenas poseían juguetes y si acaso, contaban con un parquecito infantil en el que jugaban sin orientación determinada.

Igualmente, no existía orientación para los padres pues a su vez faltaba preparación en el personal que las debía ofrecer. No se promovían planes dietéticos o nutricionales, lo que traía consigo un desperdicio de alimentos y una dieta inadecuada para los pequeños. El ingreso a la creche estaba determinado por intereses políticos o de otra índole, de los gobernantes de turno.

Todo ello nos hace llegar a la conclusión de que las creches eran una variedad de asilos o guarderías, donde los niños más que a instruirse, iban a traumatizarse.

En 1929 se aprobó el plan general de la enseñanza del Kindergarten en el país, basado en las experiencias e ideas de Froëbel, estuvo vigente hasta el curso escolar 1952-53; fue denominada Enseñanza Especial de Kindergarten y tenía dos ciclos: uno, de 4 a 5 años y otro, de 5 a 6 años. Los Kindergarten tenían como objetivo ampliar las experiencias infantiles, desarrollar hábitos de trabajo y juegos armónicos dentro del grupo.

Este plan de enseñanza estuvo influido tempranamente por los ideales educativos de la llamada Escuela Nueva o Activa, de base pragmática, que se fue perfilando durante estos años; presentaba una concepción espontánea del desarrollo con un sustento biologista fuerte en cuanto a la teoría del aprendizaje. Conducía a la individualización excesiva, se planteaba no trazar objetivos ni señalar objetivos fines y se basaba en el hecho de dejar que el niño hiciese aquello que quisiese para no forzar su libre desarrollo.

Estas instituciones perdieron prestigio, fundamentalmente, por la poca o ninguna atención que recibían del Estado, a tal extremo que en ocasiones transcurría el curso completo sin que se les suministrase algún material docente o paradocente, tan necesarios para el desarrollo de la labor de las maestras con los niños; asimismo, las maestras prácticamente no recibían capacitación que las actualizase ni asesoramiento que las preparara en los procedimientos didácticos ni en el trabajo de orientación a la familia.

No obstante, es innegable que el Kindergarten cumplió una función social en un momento histórico en el que fue el único tipo de institución que contó, en sus programas y planes de estudio, con aspectos dirigidos a la educación de los niños y niñas desde los cuatro años de edad y aunque el Estado hacía fallar sus mecanismos, constituyeron instituciones importantes para el estudio de los antecedentes de 
la educación preescolar en nuestro país.

En 1944, es instituido también el grado pre-primario en la enseñanza oficial... "con el fin de facilitar la adaptación del infante que no haya concurrido a un kindergarten, al medio escolar en que por primera vez penetra...el grado inferior será el pre-primario, que servirá de enlace entre la etapa correspondiente al kindergarten y la primaria elemental propiamente dicha."

El grado pre-primario, poseía objetivos similares a los del kindergarten, entre los que pueden destacarse el desarrollo en los niños de las capacidades requeridas para una mejor comprensión de la vida, además de intentar el desarrollo del razonamiento y su preparación para participar en la vida social. Sin embargo, a diferencia de los Kindergarten, en él predominaba una fuerte tendencia a la escolarización; el peso fundamental de sus programas lo constituía el inicio del aprendizaje como tal de la lectura, de la escritura y de las matemáticas.

En las dos últimas décadas de la república mediatizada o pseudorepública (las del 40 y del 50), cobró gran fuerza en el país la enseñanza privada, como resultado del desinterés demostrado por el Estado por la enseñanza en las escuelas públicas, ya que las medidas aplicadas por los gobiernos de turno no eran más que paliativos que no hacían otra cosa que contribuir, cada vez más, al desprestigio de este tipo de enseñanza entre los padres cubanos.

Por ello, para las clases de la pequeña y mediana burguesía aparecieron múltiples centros educacionales o instituciones religiosas que incluían la enseñanza para los niños a partir, fundamentalmente, de los cinco años.

Así, la República Neocolonial, terminó con un analfabetismo de un 23,6\%, con una escolarización del 56,4\% para las edades de 6 a 14 años, con una gran ausencia de educación especial y técnica, con más de 10 mil maestros sin escuelas y solo 15 mil alumnos en la educación superior. Para las edades preescolares, la situación era igualmente dramática: una ínfima cobertura de solo 1600 niños y niñas entre uno y seis años de edad en las 38 creches y la Casa de Beneficencia y aproximadamente 300 centros entre kindergarten y preprimarios en todo el país, generalmente privados, ubicados en La Habana y en algunas capitales de provincia, orientados por una concepción de corte pragmático y abarcando esencialmente a las edades de 5 a 6 años.

Se conoce que en las Escuelas Normales para maestros en el país se abordaban elementos teóricos de dicha tendencia pero en la práctica educativa cotidiana influía más el pensamiento pedagógico humanista martiano que los supuestos beneficios de la tendencia norteamericana, sin dejar de reconocer que algunas de las cuestiones esenciales de esta pueden tener puntos de contacto con la teoría progresista cubana desarrollada por Luz y Caballero, Félix Varela, José Martí y Enrique J. Varona como es el hecho de tener en cuenta los intereses del niño y la necesidad de hacer un proceso pedagógico más activo y práctico.

Antes del triunfo de la Revolución Cubana, existieron diversas prácticas educativas, a partir de la presencia de una educación pública y una privada. Por supuesto, era de esperar que la privada la influencia de las teorías educacionales burguesas, ya sean las que se convirtieron en tendencias pedagógicas generales o las que provenían de otros referentes de tipo, religioso, por ejemplo. En las escuelas públicas la tradición pedagógica progresista cubana estuvo más presente al menos en el espíritu 
de amor que se respiraba por numerosos maestros, aunque también la empiria estaba presente en la didáctica que se aplicaba.

\section{La revolucion (desde 1959)}

Los 56 años de república neocolonial dejaron un saldo de necesidades educativas insatisfechas. En su alegato histórico "La Historia me Absolverá", Fidel Castro, al autodefenderse en 1953 había denunciado el estado deplorable de la educación en el país.

La Revolución en el poder asumió la responsabilidad histórica de subsanar la deuda de la sociedad con el pueblo en relación con el derecho de la Educación para todos.

En 1959 se precisó que la educación era una obra priorizada de la Revolución y adquirió una dimensión humanista, martiana, participativa y democrática.

En cuanto a las edades de 0 a 6 años, el Gobierno Revolucionario se dio a la tarea de atender aquellas instituciones (creches, asilos, casas de beneficencia) que tradicionalmente estaban a cargo del estado, para lograr su perfeccionamiento, por lo que:

- Las puso en manos de personal calificado.

- Mejoró las condiciones de salud y de alimentación.

- Trasladó a los niños a edificios apropiados e higiénicos.

Las creches fueron transitando paulatinamente a hogares infantiles. En estos, cambió la concepción de los niños como "crecheros" y comenzó a considerárseles como un ser humano en condiciones de educación y desarrollo. El horario de funcionamiento se limitó entre las 7.00 a.m. y las 6.30 p.m. y contaron con un personal amplio y calificado entre las que se encontraban las maestras que conducían el proceso educativo, asesoradas y controladas por el Ministerio de Educación.

Otro tipo de institución creada en los primeros años de la Revolución fueron los Hogares-Cuna, atendidos directamente por el Ministerio de Salud Pública, cuya finalidad esencial era la de albergar, proporcionar alimentación balanceada, asistencia médica, cuidados higiénicos, formación de hábitos de vida, actividades recreativas y educación dirigida para el mejor desarrollo físico y psíquico de los niños entre 0 y 3 años, abandonados, huérfanos o remitidos por los Tribunales de Justicia.

En la clausura del acto de fundación de la Federación de Mujeres Cubanas, el 23 de Agosto de 1960, es cuando el presidente Fidel Castro expresó la necesidad de crear instituciones de nuevo tipo para el cuidado y la atención de los hijos de las madres trabajadoras e indicó que esta sería una tarea de la naciente organización.

Así, surgen los primeros Círculos Infantiles en abril de 1961, mientras que de manera natural y gradual, sin que ningún decreto o ley ordenase su desaparición, comenzó a extinguirse la beneficencia. En todos los círculos infantiles creados, se admitían niños y niñas desde los 45 días de nacidos hasta los seis años de edad de 6: 00 a.m. hasta 7:00 p.m.

La Dirección Nacional de Círculos Infantiles (DINCI) estableció contactos con otros organismos como los Ministerios de Educación y de Salud Pública, el entonces Consejo Nacional de Cultura (CNC), el Instituto Nacional de Deportes, Educación Física y Recreación (INDER) y otros, para 
orientar la atención integral del niño.

Así, en cada Círculo Infantil los niños recibían los servicios siguientes:

- Atención pedagógica.

- Atención médica.

- Atención estomatológica y oftalmológica.

- Atención de enfermería.

- Alimentación.

- Vestuario y calzado adecuados.

En el Primer Congreso de Educación y Cultura (1971) se hicieron señalamientos fundamentales, de los que no escapó la educación preescolar, que indicaba la necesidad de cambios sustanciales.

Por ello, se creael Instituto de la Infancia. Esta entidad, que surge como organismo nacional, marca un hito, una nueva etapa en el trabajo por el desarrollo de la infancia de 0 a 6 años. Su creación significó un reordenamiento estructural que unificó el trabajo de todas aquellas instituciones que se ocupaban de la educación de los niños de estas edades (Hogares Infantiles y Hogares-Cuna). Su primera presidenta, la compañera Vilma Espín, definía esta institución expresando: "El Instituto de la Infancia es la colaboración estrecha de todos los organismos en la formación de lo más preciado que tiene la sociedad, que es el niño".

Esta institución desarrolló actividades conjuntas con los Ministerios de Educación, Cultura, Salud pública, Instituto Cubano de Radio y Televisión, entre otros, así como con distintas organizaciones sociales.

Fueron creadas las Escuelas de Formación de Educadoras para Círculos Infantiles (EFECI) a las que ingresaban jóvenes con nivel medio básico ( 9 no grado). Para las educadoras no graduadas, se concibieron planes de titulación que se impartían con programas similares a los de las escuelas para educadoras.

Durante el quinquenio 1976-1980 el Instituto de la Infancia realizó un intenso trabajo en la profundización del conocimiento de las concepciones teóricas acerca del desarrollo y la educación de los niños en los primeros, y decisivos, cinco años de vida, así como, en el desarrollo de las investigaciones psicológicas y pedagógicas, sustentadas en una concepción marxista y martiana.

El perfeccionamiento continuo del Sistema Nacional de Educación fue perfilando con mayor precisión para todos los subsistemas y también para el de Educación Preescolar, la necesaria correspondencia entre el fin de la educación y la concepción del tipo de educación y de sus planes y programas.

Como resultado del derrumbe del campo socialista, la construcción de círculos infantiles se detuvo, Cuba quedó aislada y sin recursos, de tal modo, en estos centros sólo se pudo seguir atendiendo al $20 \%$ de la población infantil de cero a cinco años y en las aulas de preescolar se abarcaba aproximadamente el 73\% de la población de estas edades. Por ello se conforma un Programa Social de Atención Educativa "Educa a tu Hijo", con la participación de la familia y la comunidad para logar el desarrollo integral de los niños de cero a seis años que no asistían a instituciones infantiles. Los resultados exitosos obtenidos permitieron generalizar su aplicación a todo el país.

Actualmente, contamos con un sistema de educación preescolar perfeccionado, en el que el 
grado preescolar (cinco a seis años) forma parte del subsistema de educación preescolar, que posee un personal calificado con nivel de licenciatura en educación preescolar en más del $90 \%$ y con una cobertura de atención a la infancia del 99,5\%, de la cual el por ciento mayor se atiende por el programa Educa a tu Hijo.

El subsistema de educación preescolar, en todas sus edades e incluyendo las dos alternativas, institucional y no institucional, responden a una concepción pedagógica única, acerca de la educación de los niños, su formación y desarrollo, fundamentado en una concepción filosófica marxista y martiana.

\section{Conclusiones}

Cuando estamos atravesando el siglo XXI, Cuba puede mostrar con sano orgullo al mundo el resultado de su desarrollo cultural. Hay un patrimonio reconocible en el campo de la educación, las ciencias y la técnica y la formación de científicos y educadores; en el terreno de la creación artística y la promoción de artistas en las más diversas manifestaciones, en la edición de libros y la aparición de nuevos autores; la formación de atletas de alto rendimiento y la promoción de centenares de deportistas que han inscripto sus nombres entre los mejores del mundo, así como en otros campos del saber humano. Pero lo más importante la formación de un ciudadano, que con sus ideas, su trabajo, su espíritu de sacrifico, su solidaridad ha sido capaz de defender, hasta con su propia vida, las conquistas de la Revolución. Esto es una muestra de los logros alcanzados y de las posibilidades enormes de continuar desarrollando la educación, la ciencia, la técnica, el arte, la cultura en general.

Para acometer la búsqueda de las líneas más generales que caracterizan a la Pedagogía cubana actual, es imprescindible penetrar en toda la obra de la Revolución: en las grandes transformaciones económicas, sociales e ideopolíticas realizadas; analizar los hechos hasta su esencia y ver cómo se ha logrado revolucionar la educación en su praxis, cuáles son los métodos de desarrollo de la conciencia nacional revolucionaria que se desprenden de la estrategia pedagógica seguida por la Revolución.

La obra educacional es por su naturaleza creativa. El hecho revolucionario es la máxima fuente de inspiración para el desarrollo de la educación y la guía en la búsqueda de un camino propio, camino que habría de significar para la cultura cubana el inicio de una nueva etapa, el camino de una verdadera revolución cultural. La concepción martiana de la revolución y su proyección programática emana de un nuevo ideal de racionalidad que deviene autoconciencia cultural de nuevos sujetos. Por eso más que un acontecimiento político puro, es una empresa cultural, donde lo político, lo ético y lo estético se interpretan e implican recíprocamente.

Todo proceso de educación y formación de la personalidad en un contexto determinado nos lleva inmediatamente a la reflexión o a la consideración de la necesidad constante de cuestionamientos y eventuales cambios. Cuando hablamos de un proceso de transformación educativa nos referimos a la necesidad que tenemos, de realizar un proceso de análisis, reflexión y cuestionamiento de nuestra propia 
propuesta, para orientar y reorientar la marcha del proceso que realizamos, con el objeto de adecuarnos a legítimas y actualizadas demandas sociales.

La función pedagógica debe desarrollarse de modo que provea al niño, al adolescente y al joven un entorno que le aporte los más diversos conocimientos, que garantice la formación y el desarrollo de su personalidad integralmente y que a la vez sea un entorno emocionalmente estable y seguro. Esta condición no se da espontáneamente. Por el contrario, requiere de un trabajo de formación continua de educadores maestros y profesores, que no puede subestimarse. De ahí que necesiten más espacios compartidos para reflexionar, en los cuales puedan intercambiar acerca de sus propias dificultades, expresar sus tensiones y manifestar sus necesidades, intercambiar experiencias, resultados académicos, científicos, entre otros.

Tanto el círculo infantil,la escuela primaria como los demás centros educacionales de los diferentes niveles, hasta la universidad, son ámbitos donde los educadores ponen en juego sus representaciones, vivencias y conocimientos que determinan sus modos de pensar y actuar, lo cual da lugar a un estilo particular de diseñar y desarrollar la práctica educativa. Cada colectivo y miembro de un colectivo de educadores debe poseer pensamientos propios, que son aquellos con los cuales una persona, un grupo, se identifica profundamente, aquellos que sienten que los definen, aquellos que contribuyen a construir una identidad, pero para que esto se logre, hace falta valorar muchos otros pensamientos, otras muchas ideas, concepciones, y no considerar exclusivos los suyos, sino ser capaces de compartirlos. De ahí que sean precisamente estos pensamientos los que lo llevan a dialogar, a discutir con los demás, porque expresan sus convicciones Pensar pensamientos propios es un trabajo arduo y con consecuencias sumamente significativas.

Tomando en consideración la instrumentación de los lineamientos económicos políticos y sociales del Partido Comunista de Cuba en la esfera de la educación y con el objetivo de garantizar una formación más integral de los educandos y una mejor preparación de los maestros y profesores resaltan los motivos esenciales para impulsar las adecuaciones al proceso docente-educativo que se materializaron en todo el país a inicios del curso escolar 2014-2015, que fueron dictaminadas por la Resolución Ministerial No. 186 de 2014, como resultado de encuentros con cientos de maestros, presidentes de consejos de escuela, alumnos y directivos de Educación a todos los niveles.

Dichas medidas, tienen una mayor incidencia en la flexibilización de la organización escolar, en la posibilidad de los centros de enseñanza para organizar su horario escolar y de ese modo, responsabilizarse más con la organización del proceso docente-educativo, teniendo en cuenta sus características, las del entorno, y las potencialidades internas y externas. Esta actualización política económica y social se corresponderá con el principio de quesólo el socialismo es capaz de vencer las dificultades y preservar las conquistas de la Revolución.

La Revolución desde sus inicios asume la educación como una tarea central de la sociedad a la que dedica cuantiosos recursos materiales y humanos, lleva a cabo la transformación del aparato administrativo del Ministerio de Educación y del sistema escolar, del proceso docente educativo, 
rehaciendo la enseñanza con espíritu crítico. En el proceso se fundan instituciones y organismos de educación a tono con las nuevas realidades y se plantea además la necesidad de revolucionar la educación hasta sus cimientos. Pero lo más importante de la obra es que puede lograr revelar a los cubanos su propia naturaleza rebelde, educarlos en los conceptos de justicia, independencia, soberanía y dignidad, que es algo en que nos va la vida.

Referências

ALARCÓN ORTIZ, Rodolfo. Ministro de Educación Superior. Conferencia Especial. Congreso Pedagogía 2015 enero de 2015.

ICCP-CELEP. Proyecto de investigación para el perfeccionamiento del currículo preescolar. CELEP, La Habana, 2014.

MINED Resolución Ministerial No. 186 de 2014. Ministerio de Educación de la República de Cuba.

PCC. Proyecto de los Lineamientos de la Política Económica y Social del Partido y la Revolución.Aprobados el 18 de abril de 2011.

VELÁZQUEZ, Cobiella, Ena Elsa, Ministra de Educación. Entrevista para larevista Bohemia, 28 de agosto de 2014 .

Recebido em: 15/08/2017

Aprovado em: 07/12/2017 